\title{
Autologous bone marrow cell transplantation in acute spinal cord injury-an Indian pilot study
}

\author{
HS Chhabra, K Sarda, M Arora ${ }^{1}$, R Sharawat ${ }^{1}$, V Singh, A Nanda, GM Sangodimath and V Tandon
}

Study design: Phase- I/II, prospective, randomized, single-blind, controlled pilot study.

Primary Objective: To determine the safety and feasibility of autologous bone marrow transplantation in patients with acute spinal cord injury (SCl) via two routes of transplantation as compared with controls.

Setting: Indian Spinal Injuries Center, New Delhi.

Methods: Twenty-one subjects with acute, American Spinal Injury Association Impairment Scale (AIS) A (complete), traumatic SCI with neurological level T1-T12, were recruited and randomized into three groups of seven subjects each. Two groups underwent cell transplantation through the intrathecal or intralesional route, whereas the third served as control. Participants were assessed at baseline and followed up at 6 months and 12-months post enrollment. Safety and tolerability were evaluated by monitoring for any adverse events. Efficacy was assessed through neurological, functional and psychological evaluation, as well as through electrophysiological studies and urodynamics.

Results: Surgery was tolerated well by all participants. There were no significant adverse events attributable to the procedure. There was no significant improvement in the neurological, electrophysiological or urodynamic efficacy variables. A statistically significant improvement in functional scores as evaluated by the Spinal Cord Independence Measure and International Spinal Cord Injury Scale was observed in all groups.

Conclusions: The procedure is safe and feasible in AIS A participants with thoracic-level injuries at 12-months follow-up. No efficacy could be demonstrated that could be attributed to the procedure.

Spinal Cord (2016) 54, 57-64; doi:10.1038/sc.2015.134; published online 18 August 2015

\section{INTRODUCTION}

There is a huge base of preclinical evidence that suggests the safety and clinical efficacy of cellular therapies after spinal cord injury (SCI). ${ }^{1}$ Most of the human trials published so far have had major limitations due to flaws in design and documentation, thus severely compromising the evidence base in favor of cellular transplantation for human SCI. ${ }^{2}$ The authors had previously undertaken a prospective pilot study involving autologous olfactory mucosal transplantation in five chronic, motor, complete SCI subjects with neurological level C5-T12. ${ }^{3}$ The procedure was found to be relatively safe and feasible; however, no efficacy could be demonstrated. The failure of this and a similar study ${ }^{4}$ to replicate the efficacy previously reported by Lima et al. ${ }^{5}$ highlights the need to analyze the factors responsible for difference in outcomes of various clinical trials on human SCI. It also emphasizes the need to understand the factors that underlie the gap between the preclinical 'bench' and clinical 'bedside' findings. We hence planned to undertake another study to address the issues of 'cell population' selected, 'time of transplant or transplantation window' and the 'route of transplantation'.

Bone marrow transplantation for SCI has been the focus of attention in the past few years. There have been extensive preclinical studies demonstrating their potential role. ${ }^{1}$ Moreover, several hypotheses have been proposed to explain the potential mechanism of action of bone marrow stem cells in SCI models. First, bone marrow cells (BMCs) improve neurologic deficit by generating either neural cells or myelin-producing cells. ${ }^{6,7}$ Second, transplanted BMCs do not differentiate into neurons; rather, they work by guiding axonal regeneration by producing extracellular matrix. ${ }^{8}$ Third, transplanted BMCs promote compensatory mechanisms to reorganize the neural network and activate endogenous stem cells. ${ }^{9}$

BMCs have been transplanted by direct injection into the injured spinal cord, ${ }^{10}$ through intravenous injection, ${ }^{10,11}$ intrathecal injection ${ }^{12}$ or through injection into the spinal artery. ${ }^{10}$ Of these, the majority of recent studies have undertaken transplantation via direct injection into the lesion or via intrathecal injection, as both strategies directly target the site of injury. ${ }^{12-15}$

Hence, we undertook the study to document the safety and feasibility of administrating enriched BMCs in complete acute SCI using these two modes of cell delivery as a pilot/ prelude to a larger clinical trial using this form of therapy.

\section{MATERIALS AND METHODS}

\section{Ethics statement}

This trial was approved by the Ethics Committee of Indian Spinal Injuries Centre as well as by the Institutional Committee for Stem Cell Research and Therapy (IC-SCRT). Additional approval was also obtained from the Indian 
Council of Medical Research (ICMR). The study was registered in the ICMR database (http://ctri.nic.in/Clinicaltrials/login.php) under the number CTRI/ 2014/01/004316. Ethical guideline provisions from the Helsinki Declaration were followed. All good manufacturing practice (GMP) and good clinical practice (GCP) standards required for undertaking studies with autologous minimally manipulated cells (as used in this study) were followed to ensure quality of the cell population isolated and transplanted. Written informed consent was obtained from each subject.

\section{Patients and selection criteria}

The study was designed as a Phase- I/II (Pilot), prospective, randomized, single-blind, controlled trial over a period of 5 years. The eligibility criteria are given in Table 1. Twenty-one subjects with acute traumatic SCI (10-14 days post injury) between neurological levels T1 and T12 and complete motor and sensory loss were enrolled in this study.

All subjects underwent standardized treatment including surgical stabilization and rehabilitation. In all subjects, the procedure of surgical stabilization ( \pm decompression) was not dependent on the cell implantation procedure. Before BMC transplantation, they underwent magnetic resonance imaging and motor as well as somatosensory evoked potential (MEP and SSEP) examination.
Neurological assessment was performed by blinded assessors as per International Standards for Neurological Classification of SCI (ISNCSCI). Baseline hematological and biochemical tests were also undertaken. Table 2 summarizes the table of activity schedule of the subjects at the prescreening visit.

The enrolled subjects $(n=21)$ were randomized and divided into three groups. Randomization was carried out using a randomization box that was pre-set before the start of the study. The first group $(n=7)$ received BMCs through injection into the injured spinal cord site via durotomy (Group A). The procedure of exposure of the spinal cord, including the laminectomy, for transplantation of cells was discussed with the subjects. The second group $(n=7)$ received BMCs through the intrathecal route (Group B). The third group $(n=7)$ was the noninterventional (control) group (Group C). All assessments were conducted by a blinded assessor. The researchers administering the intervention were not blinded to the randomization schedule.

\section{Bone marrow harvesting and stem cell enrichment}

We used a point-of-care device with a closed bag system to procure enriched autologous BMCs (SmartPReP2 Bone Marrow Aspirate Concentrate System, Harvest, Plymouth, MA, USA). Before initiating the pilot study on transplantation of autologous BMCs in persons with acute SCI, a validation of

Table 1 Eligibility criteria

\begin{tabular}{ll}
\hline Inclusion criteria & Male and female subjects with traumatic spinal cord injury aged between 18 and 50 years. \\
& Acute spinal cord injury between 10 days and 14 days post injury. \\
& Traumatic spinal cord injury with neurological level of injury between T1 and T12. \\
& Complete injury (AIS A). \\
\hline Exclusion criteria & Subject whose medical condition requires mechanical ventilation. \\
& Subjects with pathological fracture. \\
& Spinal Injuries in subjects with Ankylosing Spondylitis. \\
& Subjects with pre-existing severe medical disease that would affect the outcome, like severe diabetes, rheumatoid arthritis. \\
& Subjects with psychological disorders. \\
& Pregnant subjects.
\end{tabular}

Table 2 Schedule of evaluation, assessments, subjects' visit and procedure during the study

\begin{tabular}{|c|c|c|c|c|c|c|c|}
\hline Particulars & Pre-screening & Screening & Baseline & Day of procedure & Post-op period & Month 6 & Month 12 \\
\hline Demography/medical history & $x$ & $X$ & $x$ & & & $x$ & $x$ \\
\hline Inclusion-exclusion criteria & $x$ & $x$ & $x$ & $x$ & & & \\
\hline Informed consent & $x$ & & & & & & \\
\hline Physical examination & $x$ & $x$ & $x$ & $x$ & $x$ & $x$ & $x$ \\
\hline Vital signs & $x$ & $x$ & $x$ & $x$ & $x$ & $x$ & $x$ \\
\hline Screening MRI/CT/myelogram of spinal cord & $x$ & & & & & $x$ & $x$ \\
\hline EMG of four limbs & & $x$ & & & & $x$ & $x$ \\
\hline Psychological assessment & & $x$ & & & & $x$ & $\mathrm{X}$ \\
\hline Urodynamics study & & & $\mathrm{X}$ & & & $x$ & $\mathrm{X}$ \\
\hline SSEP & & & $x$ & & & $x$ & $x$ \\
\hline MEP & & & $x$ & & & $x$ & $x$ \\
\hline Pre-operative laboratory tests & & & $x$ & & & & \\
\hline PRO questionnaire & $x$ & & $x$ & & & $x$ & $x$ \\
\hline ISNCSCI examination & $x$ & $x$ & $x$ & & & $x$ & $x$ \\
\hline $\begin{array}{l}\text { Voluntary movement in additional muscle groups not included in } \\
\text { ISNCSCI }\end{array}$ & $x$ & $x$ & $x$ & & & $x$ & $x$ \\
\hline Modified Ashworth Scale & $x$ & $\mathrm{x}$ & $\mathrm{x}$ & & & $x$ & $x$ \\
\hline WISCl & $x$ & $x$ & $x$ & & & $x$ & $x$ \\
\hline SCIM & $x$ & & $x$ & & & $x$ & $x$ \\
\hline Investigational procedure & & & & $x$ & & & \\
\hline
\end{tabular}

Abbreviations: CT, computed tomography; EMG, electromyography; MEP, motor-evoked potential; MRI, magnetic resonance imaging; ISNCSCI, International Standards for Neurological Classification of Spinal Cord Injury; PRO, patient reported outcome; SCIM, spinal cord independence measure; SSEP, somatosensory evoked potential; WISCl, Walking Index of Spinal Cord Injury.

Note: vital signs include oral body temperature, systolic and diastolic blood pressure in sitting and supine positions and pulse rate. 
the device and its stated functions was undertaken using bone marrow aspirate (BMA) from two subjects after obtaining their informed consent. A volume of $60 \mathrm{ml}$ of BMA was harvested by means of the aspiration technique from the posterior iliac crest using a 6 lumen Jamshidi-type trocar needle and 20-ml syringes, preflushed with heparin (concentration of 1000 units per $\mathrm{ml}$ ) and processed in accordance with the manufacturer's instructions. The ready-to-inject stem cell fraction had an approximate volume of 7-10 ml. Once the total and viable cell count of an aliquot of the cells was obtained, a further centrifugation of the concentrate at 1500 r.p.m. for $5 \mathrm{~min}$ at room temperature (Model No. 5804 R, Eppendorf AG, Hamburg, Germany) was undertaken to obtain a concentration of $1 \times 10^{8}$ cells $\mathrm{ml}^{-1}$.

Transplantation of BMCs through injection into the injured spinal cord site via durotomy

Although the BMA was harvested and enriched, laminectomy was performed from one vertebra above the injury site to one below using the standard procedure in order to provide sufficient access to the transplantation site. The dura mater was incised, sparing the arachnoid, which was subsequently opened separately with micro-scissors. The injury site was located using high-power microscopic magnification from normal tissue above the normal tissue below the lesion. Aliquots $(300 \mu \mathrm{l})$ of cell suspension (total volume of $1.8 \mathrm{ml}$ ) were injected into six separate positions surrounding the lesion site (two above, two below and two at the lesion site) with the injection depth of $5 \mathrm{~mm}$ from the dorsal surface and $5 \mathrm{~mm}$ lateral from the midline. A volume of $2 \times 10^{8}$ cells were injected at a rate of $300 \mu \mathrm{min}^{-1}$ using a 21 -gauge needle attached to a $1 \mathrm{ml}$ syringe. To prevent cell leakage through the injection track, the injection needle was left in position for $5 \mathrm{~min}$ after completing the injection. The dura mater and arachnoid were then closed. The muscle and skin were closed layer by layer.

\section{Transplantation by intrathecal route}

Subjects received BMCs $\left(\sim 2 \times 10^{8}\right.$ cells per $\left.1.8 \mathrm{ml}\right)$ through a lumbar puncture injection performed in the L3-4 interspace taking all aseptic precautions.

\section{Follow-up schedule}

The list of activities performed at the time of discharge, at 6-month and 12 month intervals post enrollment is given in Table 2.

\section{Safety outcome}

Safety was assessed by documenting the ascending level of SCI, neuropathic pain, aseptic meningitis, rejection of transplant, deleterious changes in MRI, changes in hematology, serum chemistry and urinalysis tests, vital signs and systemic examination.

\section{Primary efficacy end point}

Blinded assessors examined subjects for improvement of at least one American Spinal Injury Association Impairment Scale (AIS) grade by 12 months or change from baseline of $\geqslant 10$ points in total motor score.

\section{Secondary efficacy end points}

Blinded Assessors examined for any voluntary movement by additional muscle groups not included in ISNCSCI, improvements by at least 1 point in the Walking Index of Spinal Cord Injury (WISCI) scale, decrease in spasticity by one grade or more as assessed by the Modified Ashworth Spasticity scale, improvement in the American Spinal Injury Association sensory examination, improvement in SCIM score, improvements in electromyogram, SSEP and MEP, significant change in any of the variables included in urodynamic assessment, improvements in any of the variables included in Patient Reported Outcome (PRO) using ISCIS as well as changes in psychological evaluation by Beck Depression Inventory.

\section{Assessments}

The same reviewer was used throughout the study to obviate inter-rater variability. Neurological and functional evaluations were made by a trained physiotherapist and the findings reviewed by another senior.
Electrophysiological and urodynamic studies were carried out by the same neurologist and urologist, respectively, whereas psychological assessment was carried out by the same psychologist. Magnetic resonance imaging was reported by the same radiologist but the findings were validated by another radiologist.

\section{Statistical analyses}

Descriptive data are presented as mean \pm s.e.m. All statistical analyses were performed using SPSS software (SPSS Inc. Released 2007 SPSS for Windows, version 16.0., SPSS Inc., Chicago, IL, USA). Difference in mean scores between the groups was compared by means of the paired $t$-test. Missing values were excluded from analysis. $P<0.05$ was considered significant.

\section{RESULTS}

\section{Study subjects}

Twenty-one subjects met the eligibility requirements and were enrolled into this study (demographic details in Table 3 and Supplementary Table 3). The first subject was enrolled into the study on 09 February 2011 and the last on 17 November 2012.

Follow-up data at 6 months and 12 months are available for all subjects, except for three (\#006, \#011 and \#021) who did not come for the follow-up at 6 months and for two (\#006 and \#021) at 12 months after the procedure.

\section{Safety variables}

The enriched cell concentrate was reported to be sterile and had no aerobic, anaerobic or fungal elements. The concentrate was also reported to be mycoplasma and endotoxin free in all subjects. Safety data revealed that surgery was tolerated well by all subjects. There were no surgery-specific complications, except for one case, in which the sutures dehisced day 2 postoperatively. The hematological and biochemical laboratory findings were clinically insignificant postoperatively in all but one subject. The subject \#011 developed a liver abscess post transplantation. The subject opted to get transferred to another center for further treatment and was lost to follow-up at 6 months and came for the 1-year follow-up. No adverse events were reported in any other subject. Magnetic resonance imaging evaluation did not reveal any infection, hemorrhage, tumor cells or increase in cord diameter in any of the subjects.

Feasibility end points. It is an easy and simple technique. Table 4 depicts the number and characterization of the cell population transplanted in all subjects. The bone marrow harvesting and stem cell enrichment procedure consistently provided the required number of cells for transplantation.

Efficacy end points. The total American Spinal Injury Association motor scores of subjects are depicted in Figure 1. None of the subjects had significant improvement in motor score. There was no improvement in motor score of any additional muscle group not included in ISNCSCI. One participant (intervention group) improved by six points (from 0 to 6 ) and three subjects (one in the control group and two in the intervention group) improved by five points each (from 0 to 5 ) on the WISCI scale. Spasticity, as assessed by the Modified Ashworth Spasticity Scale, decreased by two grades in three subjects and by one grade in two subjects. On the contrary, it increased by one grade in two subjects. There was no statistically significant difference between the groups. The details of sensory scores for light touch and pin-prick are provided in Figure 1.

There was improvement in SCIM score from baseline visit to follow-up in all subjects (Figure 2, Supplementary Table 1). There was significant improvement as compared with baseline in all groups with regard to bladder function, bowel function, 


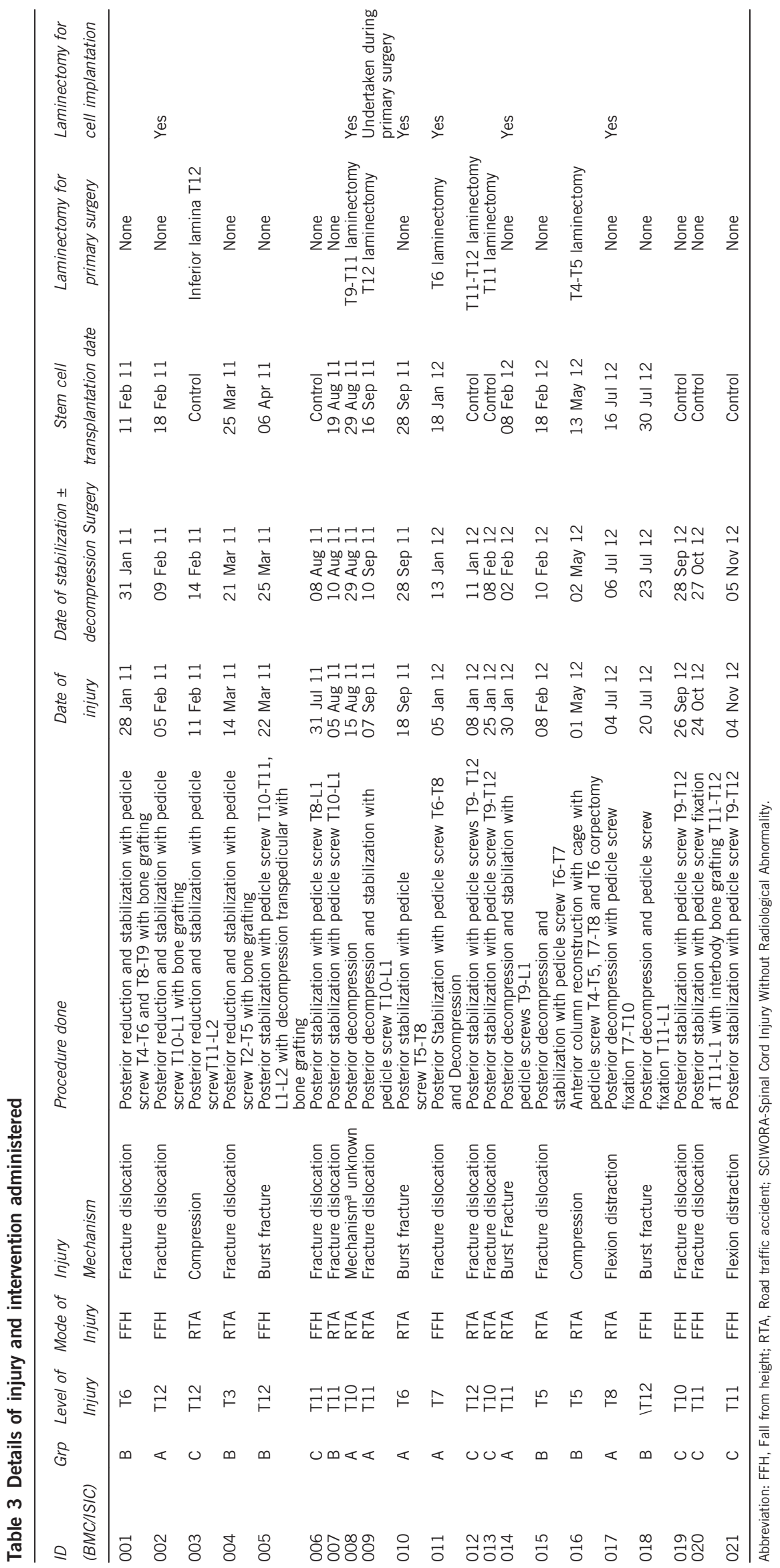




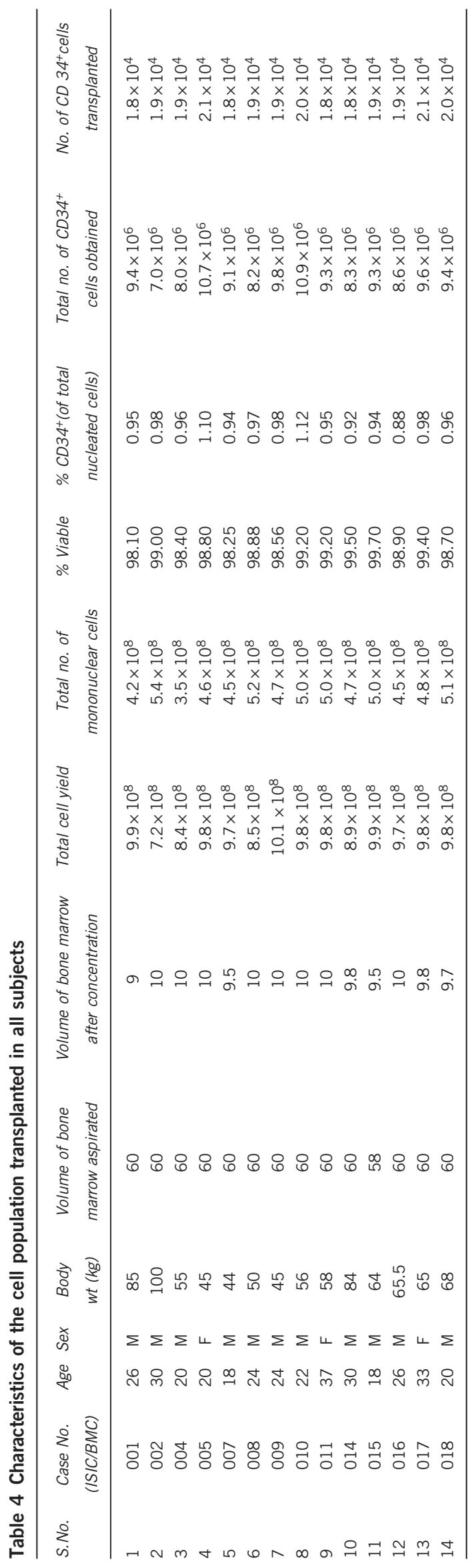

ASIA MOTOR \& SENSORY SCORES
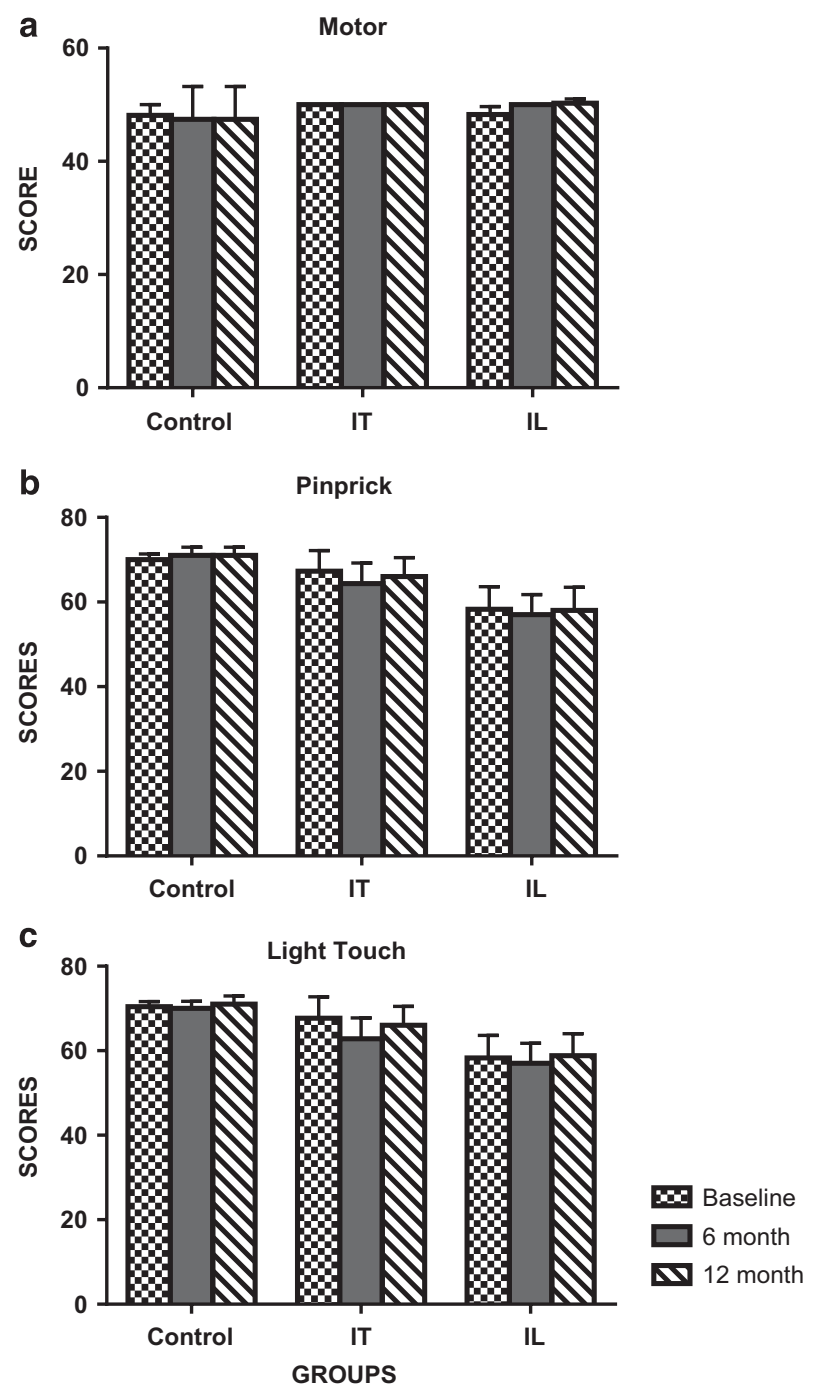

Figure 1 (a) American Spinal Injury Assessment Motor scores of all participants at baseline and at 6-monthly follow-ups. (b) International Standards for Neurological Classification of Spinal Cord Injury Sensory Examination (pin-prick) scores of all participants at baseline and at 6-monthly follow-ups. (c) International Standards for Neurological Classification of Spinal Cord Injury Sensory Examination (light touch) scores of all participants at baseline and at 6-monthly follow-ups.

injury-related pain, physical function and life impact at 6 and 12 months' follow-up on ISCIS assessment (Table 5, Supplementary Table 2). The majority of subjects chose not to answer the sexual function component of the ISCIS questionnaire; hence, data were not analyzed for this variable.

There was no change in electromyogram, SSEP or MEP in any of the subjects. There was no significant change in any variable of the urodynamic assessment in all subjects, except for reduced compliance observed in five subjects.

\section{DISCUSSION}

Despite the huge base of preclinical evidence supporting the restoration of neuronal function through cellular interventions, the clinical 


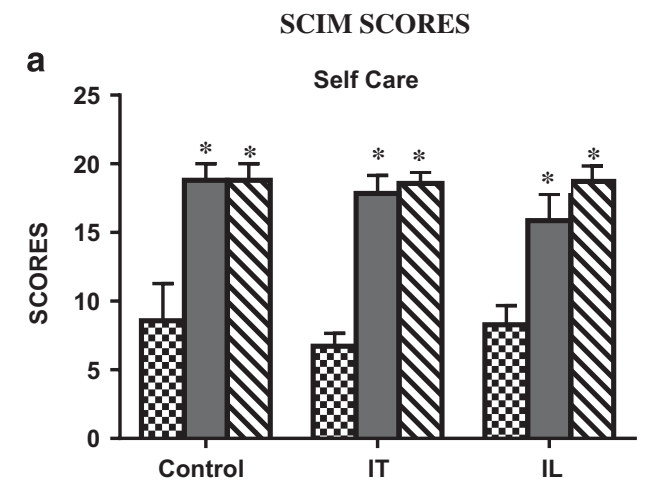

b

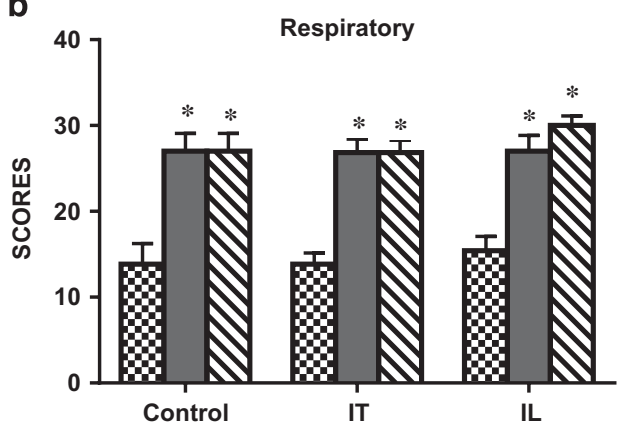

C

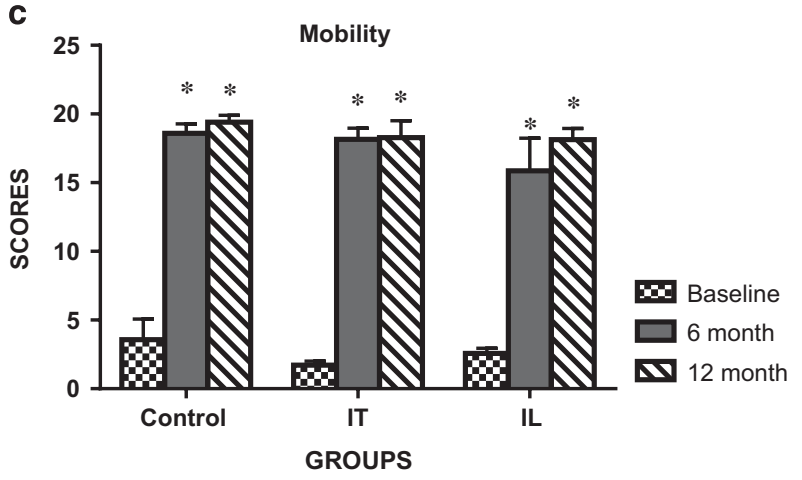

Figure 2 SCIM scores of all participants at baseline and at 6-monthly followups: (a) self-care score; (b) respiratory score; and (c) mobility score.

evidence has not been that encouraging. A huge gap still remains between the 'bench' and the 'bedside' and needs to be bridged. ${ }^{16}$ This may be due to the inherent limitations of the preclinical studies as their conclusions are dependent upon the mechanism of injury and the animal model used. ${ }^{2}$ Also, factors such as cell population used, timing of intervention, patient selection criteria, spontaneous recovery confounding the interpretation of results, poor trial design and lack of standardized outcome measures underlie the low level of clinical evidence base supporting the efficacy of cellular transplantation in case of SCI. ${ }^{2,17}$ The trial was thus designed to address these factors with an aim to provide robust, standardized and validated data regarding the potential of stem cell interventions in case of SCI.

As compared with incomplete SCI subjects (AIS B, AIS C and AIS D), motor complete SCI subjects (AIS A) have fairly limited and predictable neurological recovery. Most of the spontaneous neurological recovery in AIS A subjects is likely to occur within the Zone of Partial Preservation. ${ }^{18}$ Hence, only AIS A subjects were included in the study.
The potential of regeneration and recovery of the spinal cord may reduce with time. ${ }^{1,17}$ Hence, even though it is preferable to recruit chronic SCI subjects in such a trial (as any potential for spontaneous recovery would have plateaued by that time), we chose to do this study in acute SCI patients.

Preclinical studies suggest that transplants are more likely to survive if the procedure is undertaken during the subacute phase (10-14 days post injury) as the secondary cascade events in the acute phase of injury may affect the survival of the graft. ${ }^{19,20}$ In a clinical trial Yoon et al. ${ }^{21}$ have reported a better outcome following cell transplant in their acute and subacute injury group as compared with the chronic injury group. Also, as per guidelines for the conduct of clinical trials for SCI as developed by the ICCP Panel, any therapy initiated within the acute phase of injury (up to $\sim 72 \mathrm{~h}$ post injury) would need a very large number of subjects to identify significant differences due to the intervention as a result of high probability (20\%) of spontaneous recovery in AIS A subjects at this stage. ${ }^{22}$ In the subacute phase of injury, the probability of spontaneous recovery in case of AIS A subjects reduces to $10 \%$, leading to a reduction in the number of subjects required to detect a potentially significant benefit. Hence, we chose to undertake the intervention in the subacute (10-14 days post injury) phase of injury. If during manipulation of cervical spinal cord regions of critical healthy spinal cord tissue rostral to the injury are compromised, it may lead to severe adverse events. ${ }^{22}$ Thus, for this study subjects with cervical neurological level lesions were not included.

The study design was in conformance with the 'Guidelines for the conduct of clinical trials for spinal cord injury' as developed by the International Campaign for Cures of SCI Paralysis panel. ${ }^{22}$

Bone marrow transplantation for SCI has been the focus of attention in the past few years. There have been extensive preclinical studies that have demonstrated their potential role. Transplanted BMCs were found to improve neurological deficit in CNS injury models by generating neural cells or myelin-producing cells. ${ }^{6}$ The use of BMCs for stem cell therapy in SCI subjects has more advantages compared with embryonic stem cells; therefore, the cell population has been used more widely. ${ }^{1}$ Extensive scientific data on BMCs are also available from wide-ranging experiences in bone marrow transplantation for hematological diseases. ${ }^{23}$ Bone marrow stem cell-based therapy is not associated with carcinogenesis, which is an adverse event documented in case of embryonic stem cell therapy. ${ }^{24}$ It is also possible to use these cells for allogenic transplantation as there is little risk of immunological rejection or graft-versus-host reactions. ${ }^{25}$

The safety of transplanted unmanipulated autologous bone marrow in subjects with SCI has been reported by Sykova et al. ${ }^{26}$ However, the authors could not confirm that the observed beneficial effects were due to cellular intervention. ${ }^{26}$ Other groups have transplanted enriched or expanded bone marrow mesenchymal stem cells in SCI subjects either alone or in combination with CD34+ cells or GMCSF. ${ }^{21,25}$ However, because of various limitations of these studies, the outcomes reported are inconclusive. ${ }^{2}$

This study was initially planned to transplant the Ficoll-purified mononuclear cell population as used in other studies. However, because of regulatory constraints in India that did not allow the use of a Ficoll-purified population, an enriched BMC population was transplanted. Before undertaking the transplantation, a validation study was undertaken by us where the enriched bone marrow population was analyzed for $\mathrm{CD} 34+$ cell and mononuclear cell percentage. These were found to be comparable to that reported for Ficoll-purified cells. ${ }^{27}$ The sterility of processed cells was also established by us. 
Table 5 ISCIS $^{\text {a }}$ scores at baseline, 6-month and 12-month follow-up

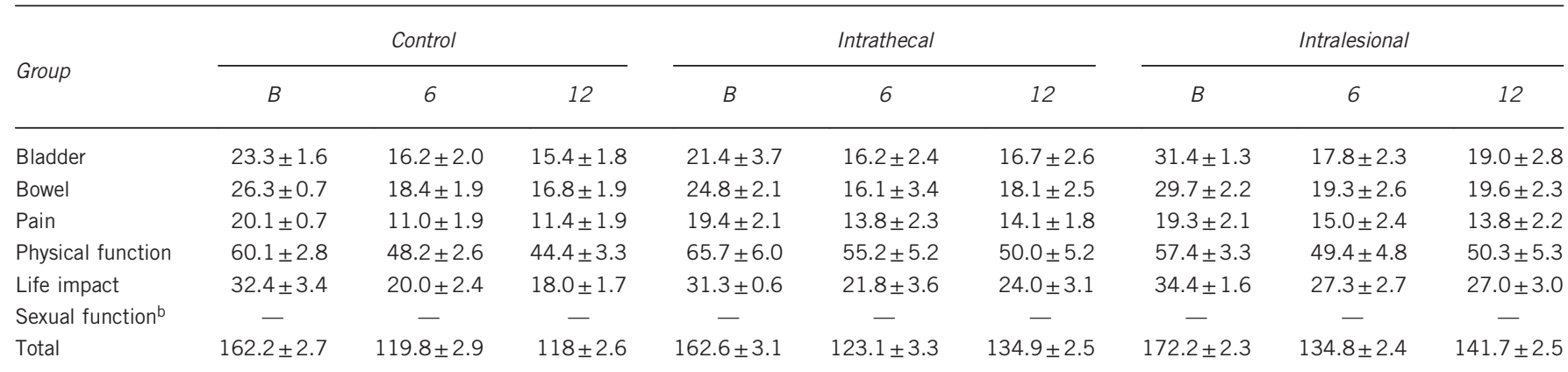

Abbreviation: ISCIS, International Spinal Cord Injury Scale.

ascores \pm s.e.m.

${ }^{\mathrm{b}}$ Not analyzed because of insufficient data

The procedure was found to be safe, as revealed by our 12-month follow-up findings. There was no incidence of infection/meningitis. There was one incidence of liver abscess in a subject who received the cells via intrathecal transplantation. The exact cause of the liver abscess could not be determined. The prescreening and screening assessments of the subject had been normal and the transplanted material was found to be negative for aerobic and anaerobic bacteria, mycoplasma and fungus. The subject had opted to get transferred to another center for further treatment and the abscess had completely resolved. The subject was lost to follow-up at 6 months. At the 12-month follow-up visit, the liver abscess was documented by the other center as a fungal infection. As the transplanted material was reported to be sterile, the cause of the infection may have been factors other than the transplanted material. The subject had been referred to us from another hospital where the subject had received primary treatment for the SCI. This could be a case of hospital-acquired or coincidental infection.

Zariffa et al. ${ }^{28}$ suggested that deterioration of three or more thoracic sensory levels or loss of upper-extremity motor function are rare events and may be used to track the safety of therapeutic intervention in early-phase acute SCI clinical trials. ${ }^{28}$ The absence of this in our subjects also suggests the safety of the intervention. A longer follow-up is being undertaken to establish long-term safety.

The stringent inclusion-exclusion criteria resulted in a slow recruitment of subjects. This problem had also been faced by another similar study with similar inclusion- exclusion criteria. ${ }^{4}$ Another factor that slowed down the recruitment in our study was that subjects, who initially showed interest in our study, later opted for one of the numerous other centers in the country offering some form of cellular transplantation 'cure'.

Single blinding of assessors may have led to decreased compliance to trial follow-up visits by the control subjects. This may have resulted in two subjects being lost to follow-up (at both 6- and 12-month visits) in the control group. Only one subject was lost to follow-up from the interventional groups at the 6-month visit but was recovered at the 12-month visit.

Our study established the feasibility of the procedure. It was possible to aspirate bone marrow from the patient and transplant it within $2 \mathrm{~h}$ of aspiration. The cell population studied in our study was an enriched BMC population and not a Ficoll-purified mononuclear cell population as reported by others. The data provided by our study regarding the composition of the cell population transplanted are of importance as the data provide leads to correlating the number of cells and transplanted cell population with clinical findings in the follow-up visits. This would also be useful for standardizing the dose of transplanted cells in future studies.
There was no significant improvement in the primary efficacy variable, that is, the American Spinal Injury Association motor score of the subjects. Such an outcome corresponds to the natural history of complete SCI. ${ }^{17}$ The study was just a pilot study and a larger study with a longer follow-up is required to determine the efficacy of the procedure.

There was also no significant improvement in the secondary efficacy variables other than SCIM or ISCIS. The statistically significant improvement in functional examination (SCIM) scores without any significant changes in neurological (American Spinal Injury Association motor or sensory score) or electrophysiological (electromyogram, SSEP or MEP) outcomes suggests that the improvements in SCIM may have been because of compensation and exploitation of neuronal plasticity by functional training and not because of regeneration. Curt et al. ${ }^{17}$ have postulated this mechanism for improvement in activities of daily living of subjects that occurs without any change in neurological condition. The functional improvement could be responsible for the statistically significant improvement in scores of physical function and life impact (as demonstrated through the ISCIS questionnaire). The improvement in scores of bladder and bowel function could be due to the bladder and bowel training.

The findings of our study differ from those of other human trials undertaking transplantation of autologous BMCs in SCI subjects published so far ${ }^{13,15,21,25,26,29}$ in that no efficacy could be demonstrated in our study that could be attributed to the procedure. This disparity may be attributed to our stringent trial design with only AIS A subjects, type of cell population transplanted and validated outcome measures used by us to analyze the effect of BMC transplantation in persons with acute SCI. Some of the previous studies had also enrolled incomplete SCI subjects and thus any improvement could have been due to the natural history of the disease. Further, assessors were not blinded in most of these studies and many also lacked appropriate control groups.

Recent publications on the mechanisms involved in repair and regeneration post SCI provide valuable insights regarding potential barriers to regeneration after SCI. ${ }^{24}$ These need to be addressed by scientists and clinicians to define new strategies for achieving repair. Basic scientific research should be directed toward providing a rational basis for tailoring specific combinations of clinical therapies to different types of SCI. Functional regeneration should be the primary goal of any approach being tested and it is important that this be tested by scientifically validated and universally accepted outcome measures and tools.

Because of the involvement of multiple cell types and the complexity of SCI, it is becoming increasingly clear that a single approach as 
used in our study may not be successful in achieving SCI repair. ${ }^{2}$ A multifactorial approach involving inhibition of secondary cascade events, molecular therapy, cell transplantation, bioactive scaffolding matrix, growth factor supplementation and scar removal is required to address this situation. Multicentric studies involving a standardized and validated approach, a stringent trial design with appropriate outcome measures and rehabilitation protocol are a must to understand and achieve the potential of such combinatorial strategies (including cellular therapy) in case of SCI.

The following could be the limitations of this study:

- The small sample size may not be enough to conclude on the efficacy of the study.

- The outcomes may have been affected by an inappropriate number of transplanted cells.

\section{CONCLUSIONS}

The procedure of BMC transplantation is relatively safe and feasible in AIS A subjects with thoracic-level injuries at 12-month follow-up. A full follow-up of 5 years in all 21 subjects will further reinforce the safety of the procedure. No efficacy could be demonstrated that could be attributed to the procedure. However, it may not be possible to conclude on the efficacy of the procedure because of the limitations of the study.

\section{CONFLICT OF INTEREST}

The authors declare no conflict of interest.

\section{ACKNOWLEDGEMENTS}

The work was jointly funded by the Indian Council of Medical Research and Indian Spinal Injuries Centre. We acknowledge the contribution of Dr Nishit Patel and Dr Rajat Mahajan for support for conducting this study. We thank the Indian Council of Medical research for making valuable suggestions to the study protocol. We also convey our appreciation to Dr AK Sahani for conducting the electrophysiological tests for all participants, Mrs Chitra Kataria for reviewing the neurological and functional assessments, Dr Divya Parashar for psychological assessments and Dr Vandana Phadke for statistical analyses.

\section{DATA ARCHIVING}

There were no data to deposit.

1 Li J, Lepski G. Cell transplantation for spinal cord injury: a systematic review. Biomed Res Int 2013; 2013: 32.

2 Chhabra HS, Sarda K. Stem cell therapy in spinal trauma: does it have scientific validity? Indian J Orthop 2015; 49: 56-71.

3 Chhabra HS, Lima C, Sachdeva S, Mittal A, Nigam V, Chaturvedi D et al. Autologous olfactory [corrected] mucosal transplant in chronic spinal cord injury: an Indian Pilot Study. Spinal Cord 2009; 47: 887-895.

4 Mackay-Sim A St, John JA. Olfactory ensheathing cells from the nose: clinical application in human spinal cord injuries. Exp Neurol 2011; 229: 174-180.

5 Lima C, Pratas-Vital J, Escada P, Hasse-Ferreira A, Capucho C, Peduzzi JD. Olfactory mucosa autografts in human spinal cord injury: a pilot clinical study. J Spinal Cord Med 2006; 29: 191-203.

6 Chopp M, Zhang XH, Li Y, Wang L, Chen J, Lu D et al. Spinal cord injury in rat: treatment with bone marrow stromal cell transplantation. Neuroreport 2001; 11: 3001-3005.
7 Sasaki M, Honmou O, Akiyama Y, Uede T, Hashi K, Kocsis JD. Transplantation of an acutely isolated bone marrow fraction repairs demyelinated adult rat spinal cord axons. Glia 2001; 35: 26-34.

8 Gu W, Zhang F, Xue Q, Ma Z, Lu P, Yu B. Transplantation of bone marrow mesenchymal stem cells reduces lesion volume and induces axonal regrowth of injured spinal cord. Neuropathology 2010; 30: 205-217.

9 Burns AS, Lee BS, Ditunno JF, Tessler A. Patient selection for clinical trials: the reliability of the early spinal cord injury examination. J Neurotrauma 2003; 20: 477-482.

10 Geffner LF, Santacruz P, Izurieta M, Flor L, Maldonado B, Auad AH et al. Administration of autologous bone marrow stem cells into spinal cord injury patients via multiple routes is safe and improves their quality of life: comprehensive case studies. Cell Transplant 2008; 17: 1277-1293.

11 Sahni V, Kessler JA. Stem cell therapies for spinal cord injury. Nat Rev Neurol 2010; 6: 363-372.

12 Kishk NA, Gabr H, Hamdy S, Afifi L, Abokresha N, Mahmoud H et al. Case control series of intrathecal autologous bone marrow mesenchymal stem cell therapy for chronic spinal cord injury. Neurorehabil Neural Repair 2010; 24: 702-708.

13 Bhanot Y, Rao S, Ghosh D, Balaraju S, Radhika CR, Satish Kumar K V. Autologous mesenchymal stem cells in chronic spinal cord injury. Br J Neurosurg 2011; 25: 516-522.

14 Karamouzian S, Nematollahi-Mahani SN, Nakhaee N, Eskandary H. Clinical safety and primary efficacy of bone marrow mesenchymal cell transplantation in subacute spinal cord injured patients. ClinNeuro/Neurosurg 2012; 114: 935-939.

15 Jarocha D, Milczarek O, Kawecki Z, Wendrychowicz A, Kwiatkowski S, Majka M. Preliminary study of autologous bone marrow nucleated cells transplantation in children with spinal cord injury. Stem Cells Trans/ Med 2014; 3: 395-404.

16 Hewson SM, Fehlings LN, Messih M, Fehlings MG. The challenges of translating stem cells for spinal cord injury and related disorders: what are the barriers and opportunities? Expert Rev Neurother 2013; 13: 143-150.

17 Liverman CT, Altevogt BM, Joy JE, Johnson RT. Spinal Cord Injury: Progress, Promise, and Priorities. National Academies Press, Washington D.C., USA: 2005.

18 Curt A, Van Hedel HJA, Klaus D, Dietz V. Recovery from a spinal cord injury: significance of compensation, neural plasticity, and repair. J Neurotrauma 2008; 25: 677-685.

19 Hofstetter CP, Schwarz EJ, Hess D, Widenfalk J, EI Manira A, Prockop DJ et al. Marrow stromal cells form guiding strands in the injured spinal cord and promote recovery. Proc Natl Acad Sci USA 2002; 99: 2199-2204.

20 Okano H, Ogawa Y, Nakamura M, Kaneko S, Iwanami A, Toyama Y. Transplantation of neural stem cells into the spinal cord after injury. Semin Cell Dev Biol 2003; 14: 191-198.

21 Yoon SH, Shim YS, Park YH, Chung JK, Nam JH, Kim MO et al. Complete spinal cord injury treatment using autologous bone marrow cell transplantation and bone marrow stimulation with granulocyte macrophage-colony stimulating factor: Phase I/II clinical trial. Stem Cells 2007; 25: 2066-2073.

22 Tuszynski MH, Steeves JD, Fawcett JW, Lammertse D, Kalichman M, Rask C et al. Guidelines for the conduct of clinical trials for spinal cord injury as developed by the ICCP Panel: clinical trial inclusion/exclusion criteria and ethics. Spinal Cord 2007; 45 : 222-231.

23 Wright KT, El Masri W, Osman A, Chowdhury J, Johnson WEB. Concise review: bone marrow for the treatment of spinal cord injury: mechanisms and clinical applications. Stem Cells 2011; 29: 169-178.

24 Horner P, Gage F. Regenerating the damaged central nervous system. Nature 2000; 407: 963-970.

25 Ichim TE, Solano F, Lara F, Paris E, Ugalde F, Rodriguez JP et al. Feasibility of combination allogeneic stem cell therapy for spinal cord injury: a case report. Int Arch Med 2010; 3: 30 .

26 Syková E, Homola A, Mazanec R, Lachmann $H$, Konrádová SL, Kobylka $\mathrm{P}$ et al. Autologous bone marrow transplantation in patients with subacute and chronic spinal cord injury. Cell Transplant 2006; 15: 675-687.

27 Dedeepiya VD, Rao YY, Jayakrishnan G a, JKBC Parthiban, Baskar S, Manjunath SR et al. Index of CD34+ cells and mononuclear cells in the bone marrow of spinal cord injury patients of different age groups: a comparative analysis. Bone Marrow Res 2012; 2012: 8.

28 Zariffa J, Kramer JLK, Fawcett JW, Lammertse DP, Blight AR, Guest J et al. Characterization of neurological recovery following traumatic sensorimotor complete thoracic spinal cord injury. Spinal Cord 2011; 49: 463-471.

29 Amr SM, Gouda A, Koptan WT, Galal AA, Abdel-Fattah DS, Rashed LA et al. Bridging defects in chronic spinal cord injury using peripheral nerve grafts combined with a chitosan-laminin scaffold and enhancing regeneration through them by co-transplantation with bone-marrow-derived mesenchymal stem cells: case series of 14 patient. J Spinal Cord Med 2014; 37: 54-71.

Supplementary Information accompanies this paper on the Spinal Cord website (http://www.nature.com/sc) 